\title{
On measuring the cosmic-ray production rate in supernova remnant shocks by polarized Balmer line emission
}

\author{
Jiro Shimoda* \\ Frontier Research Institute for Interdisciplinary Sciences, Tohoku University, \\ Astronomical Institute, Tohoku University, Sendai 980-8578, Japan \\ E-mail: i-shimodadastr.tohoku.ac.ip
}

\section{Yutaka Ohira}

Department of Earth and Planetary Science, The University Tokyo, 7-3-1, Hongo, Bunkyo-ku, Tokyo 113-0033, Japan

E-mail: v.ohiradeps.s.u-tokvo.ac.jp

\section{Ryo Yamazaki}

Depertment of Physics and Mathematics, Aoyama-Gakuin University, Sagamihara, Kanagawa 252-5258, Japan

E-mail: ryodphys.apyama.ac.jp

\section{J. Martin Laming}

Space Science Division, Naval Research Laboratory, Code 7684, Washington DC 20375, USA

E-mail: Lamingenrl.navy.mil

\section{Satoru Katsuda}

Graduate School of Science and Engineering, Saitama University, 255 Shimo-Ohkubo, Sakura, Saitama 338-8570, Japan

E-mail: katsudadphys.saitama-u.ac.jp

Linearly polarized Balmer line emissions from supernova remnant shocks are studied taking into account the energy loss of the shock owing to the production of nonthermal particles. The polarization degree depends on the downstream temperature and the velocity difference between upstream and downstream regions. The former is derived once the line width of the broad component of the $\mathrm{H} \alpha$ emission is observed. Then, the observation of the polarization degree tells us the latter. At the same time, the estimated value of the velocity difference independently predicts the apparent downstream temperature on the assumption that the shock is adiabatic. If the actually observed downstream temperature is lower than the apparent one, there is a missing thermal energy which is consumed for particle acceleration. It is shown that a larger energy loss rate leads to more highly polarized $\mathrm{H} \alpha$ emission. In comparison of actual observations, our result implies a sizable energy loss rate for the SN 1006 shock.

36th International Cosmic Ray Conference -ICRC2019-

July 24th - August 1st, 2019

Madison, WI, U.S.A.

\footnotetext{
* Speaker.
} 


\section{Introduction}

The energy density of cosmic-rays (CRs), measured around the Earth at about $1 \mathrm{GeV}$ is comparable to the energy densities of the main components of our galaxy such as stellar light, thermal gas and magnetic field pressure. In our galaxy, the pressures of the magnetic field and CRs support matter against its own weight in the galactic gravitational potential (e.g. [W]). Moreover, the CRs ionize dense, cold molecular gases $(\sim 10 \mathrm{~K})$ that are star-formation sites. Since the ionization degree of gas characterizes the gas dynamics via the frozen-in magnetic field, the CRs may regulate star-formation (e.g. [■], [], 团]). Then, supernovae originated from massive stars inject energy so that the multiphase structure of interstellar medium and thus star-formation in our galaxy are maintained ([[5]).

The origin of CRs is a long-standing unresolved issue in Astrophysics. CRs with energy up to $3 \mathrm{PeV}$ are usually called 'Galactic CRs'. It is believed that the collisionless shock waves of supernova remnants (SNR) are the best candidates for the origin of Galactic CRs. The 'collisionless' aspect means that the shock transition occurs on a length scale much shorter than that associated with a particle mean free path to Coulomb scattering. Therefore, thermal equilibrium is much less strongly enforced. If roughly a tenth of supernova the explosion energy is consumed for the CR acceleration, the energy density measured around the Earth can be explained (e.g. [6]). Thus, the physics of particle acceleration is one of the most important issues of collisionless shocks.

An important concern is to specify the density of accelerated particles in SNR shocks, a necessary step towards confirming the shocks as the main source of Galactic CRs. In addition, it allows us to quantify the back reaction of accelerated particles on the background shock structure. If the amount of accelerated particles is significant, in other words, if a large fraction of shock kinetic energy goes to non-thermal particles resulting from the acceleration, the downstream temperature becomes considerably lower than in the case of an adiabatic shock, i.e., there is some missing thermal energy. (e.g. [ $[\mathbb{Z}, \mathbb{8}, \mathbb{Q}]$ ). Therefore, if we measure both the downstream temperature $\left(T_{\text {down }}\right)$ and the shock velocity $\left(V_{\mathrm{sh}}\right)$ independently, we can estimate the energy loss rate. In order to do this, we define $k_{\mathrm{B}} T_{\mathrm{RH}}=\frac{3}{16} \mu m_{\mathrm{p}} V_{\mathrm{sh}}{ }^{2}\left(k_{\mathrm{B}}\right.$ is the Boltzmann constant and $\mu$ is the mean molecular weight), which is the adiabatic downstream temperature predicted by Rankine-Hugoniot relation in the strong shock limit without CR acceleration (i.e. adiabatic shocks). Then, the energy-loss rate is defined as ([ए]]),

$$
\eta \equiv \frac{T_{\mathrm{RH}}-T_{\mathrm{down}}}{T_{\mathrm{RH}}}
$$

Observations of SNR RCW 86 give an example. The shock velocity is measured by the proper motion of an $\mathrm{H} \alpha$ filament as $V_{\mathrm{sh}} \approx 1800 \mathrm{~km} \mathrm{~s}^{-1}$, which gives $k_{\mathrm{B}} T_{\mathrm{RH}} \approx 4 \mathrm{keV}(\mu / 0.62)\left(V_{\mathrm{sh}} / 1800 \mathrm{~km} \mathrm{~s}^{-1}\right)^{2}$ ([ए]]). On the other hand, the downstream temperature is derived from spectroscopy of the $\mathrm{H} \alpha$ emission as $k T_{\text {down }} \approx 2 \mathrm{keV}$ ([[]]). Combining these observations, we obtain $\eta \approx 0.5$. Unfortunately, to evaluate the shock velocity from the proper motion measurements, we need a distance to the SNR with high accuracy, which is often hard in astronomy.

In the latest development, we showed that the polarimetry of $\mathrm{H} \alpha$ can be a novel diagnostic for the energy loss rate independently of the distance (see [12]). Recently, [[13] discovered linearly polarized $\mathrm{H} \alpha$ emission with $2.0 \pm 0.4$ per cent polarization degree in the north-west region of the 
young SNR, SN 1006. In laboratory experiments, linearly polarized $\mathrm{H} \alpha$ emission from hydrogen atoms excited by electron beams has been measured with $\sim 40$ per cent polarization degree [14]. The electron beam, which collides with hydrogen atoms from only one direction, behaves as a quantization axis of the orbital angular momentum of the bound electron in excited hydrogen atoms. The bound electrons lose their energy and total angular momentum owing to the spontaneous transition, and emit photons. The polarization of the photon is then determined to be linear or circular by the variation of the orbital angular-momentum component along with the beam direction. The linearly polarized intensity becomes largest when viewed from the direction orthogonal to the beam. For BDS, charged hot particles impact the cold hydrogen atoms from various directions in the downstream region. In the rest frame of the cold hydrogen atoms (i.e. the upstream frame), the colliding charged particles are seen as a mildly-collimated beam. Therefore, this anisotropy eventually causes a net polarization of the line emission, with the polarization degree of a few percent. If the SNR shock efficiently accelerates CRs, then they can escape the shock, carrying away significant energy. As a result, the downstream temperature becomes lower, yielding larger anisotropy of the particle velocity downstream. In [12], we studied the linearly polarized Balmer line emissions from the downstream region of SNR shocks losing their thermal energy. We showed that a higher energy loss rate causes higher polarization degree. The polarization degree of the line emission is determined by the anisotropy of the velocity distribution of charged particles (i.e. collimation of incident beam), which is given by the downstream temperature and the upstream fluid velocity. We can measure the downstream temperature from the width of $\mathrm{H} \alpha$ line, whereas the downstream fluid velocity is derived from the polarization measurements. Since the shock velocity $V_{\mathrm{sh}}$ relates $T_{\mathrm{RH}}$ by Rankine-Hugoniot relation, we can obtain the energy loss rate $\eta$ without measuring the SNR distance.

The polarization degree of $\mathrm{H} \alpha$ depends on the optical depth of the Lyman series line ( $\operatorname{Ly} \beta$, $\operatorname{Ly} \gamma$ and so on). This is because when hydrogen atoms in the $3 p$ state emit Ly $\beta$, this emitted Ly $\beta$ is absorbed by other hydrogen atoms, the atom is re-excited to the $3 p$ state, and eventually the re-excited atom emits $\mathrm{H} \alpha$. In [ㅁ] ], we considered only the optically thin limit and thick limit of the Lyman line, although it has been pointed out that the depth is of intermediate thickness in realistic SNRs (e.g. [대]). Indeed, by constructing a sophisticated radiative line transfer model of SNR shock, [1]] confirmed the intermediate optical thickness. Thus, in this paper, combining the results of [ए]] and [ए6], we estimate more a realistic polarization degree of the Balmer lines (especially $\mathrm{H} \alpha$ ). Note that throughout this paper, we consider only 'narrow' component of $\mathrm{H} \alpha$ that is emitted by hydrogen atoms experiencing no charge exchange reactions (see [ए2] for detail).

\section{Model Calculations}

In order to evaluate the effects of $\mathrm{Ly} \beta-\mathrm{H} \alpha$ conversion upon the $\mathrm{H} \alpha$ polarization degree in realistic shocks, we use the results of atomic population calculation by [16]. In the model of [ए6], the SNR shock is set to be plane-parallel and axially-symmetric about the $z$-axis. This geometry is the same as [12]. Thus, the emission coefficient of the total intensity of $\mathrm{H} \alpha$ (Stokes $I$ of $\mathrm{H} \alpha$ ) depends only on $z$,

$$
j_{v, \mu}^{I}(z)=\frac{h v}{4 \pi}\left\{n_{\mathrm{H}, 3 \mathrm{~s}} A_{3 \mathrm{~s}, 2 \mathrm{p}} \phi_{v, \mu}^{3 \mathrm{~s}, 2 \mathrm{p}}+n_{\mathrm{H}, 3 \mathrm{p}} A_{3 \mathrm{p}, 2 \mathrm{~s}} \phi_{v, \mu}^{3 \mathrm{p}, 2 \mathrm{~s}}+n_{\mathrm{H}, 3 \mathrm{~d}} A_{3 \mathrm{~d}, 2 \mathrm{p}} \phi_{v, \mu}^{3 \mathrm{~d}, 2 \mathrm{p}}\right\},
$$


where $h$ is Planck's constant, $n_{\mathrm{H}, j}$ is the number density of hydrogen atom in the state $j, A_{k, j}$ is the spontaneous transition rate from the state $k$ to $j$ and $\phi_{v, \mu}^{k, j}$ is the line profile function for the transition from $k$ to $j$, respectively. The frequency of photon is $v$ and $\mu=\cos \theta$ denotes the direction cosine of the photon, that is the wave vector of photon makes an angle $\theta$ with the $z$-axis. In the following, we fix our line of sight along the $y$-axis $(\mu=0)$. Supposing the optically thin limit for the Balmer lines, we obtain the total specific intensity of $\mathrm{H} \alpha$ as

$$
I_{v, 0}(z)=j_{v, 0}^{I}(z) L
$$

where $L$ is the path length along the line of sight. For the emission coefficient of polarized intensity (Stokes $Q$ ), we assume that the radiative excitation ( $\mathrm{Ly} \beta-\mathrm{H} \alpha$ conversion) and cascades from higher levels yield completely unpolarized $\mathrm{H} \alpha$ for simplicity, ${ }^{1}$ that is, the polarized intensity only originates from the collisional excitation. Then, we approximately obtain the emission coefficient of polarized intensity of $\mathrm{H} \alpha$ as

$$
j_{v, \mu}^{Q}(z) \approx \frac{h v}{4 \pi}\left\{n_{\mathrm{H}, 1 \mathrm{~s}} C_{1 \mathrm{~s}, 3 \mathrm{~s}} \phi_{v, \mu}^{3 \mathrm{~s}, 2 \mathrm{p}}+n_{\mathrm{H}, 1 \mathrm{~s}} C_{1 \mathrm{~s}, 3 \mathrm{p}} \phi_{v, \mu}^{3 \mathrm{p}, 2 \mathrm{~s}}+n_{\mathrm{H}, 1 \mathrm{~s}} C_{1 \mathrm{~s}, 3 \mathrm{~d}} \phi_{v, \mu}^{3 \mathrm{~d}, 2 \mathrm{p}}\right\} \sqrt{1-\mu^{2}} P,
$$

where $\sqrt{1-\mu^{2}} P$ is the polarization degree of $\mathrm{H} \alpha$ for the optically thin limit of the Lyman lines. Note that $\sqrt{1-\mu^{2}} P$ is equal to the ratio of equation (19) to equation (20) in [ㄷ]]. The collisional excitation rate for the transition from $j$ to $k$ is $C_{j, k}$. Note that for the calculation of $j_{v, \mu}^{Q}$, we omit the excitation via the charge exchange reaction. Thus, the Stokes $Q$ along the line of sight is given as

$$
Q_{v, 0}(z)=j_{v, 0}^{Q}(z) L
$$

Then, the observed polarization degree of $\mathrm{H} \alpha$ is

$$
\Pi_{0}(z)=\frac{\int Q_{v, 0} \mathrm{~d} v}{\int I_{v, 0} \mathrm{~d} \nu} \approx P \frac{n_{\mathrm{H}, 1 \mathrm{~s}}\left(C_{1 \mathrm{~s}, 3 \mathrm{~s}}+C_{1 \mathrm{~s}, 3 \mathrm{p}}+C_{1 \mathrm{~s}, 3 \mathrm{~d}}\right)}{n_{\mathrm{H}, 3 \mathrm{~s}} A_{3 \mathrm{~s}, 2 \mathrm{p}}+n_{\mathrm{H}, 3 \mathrm{p}} A_{3 \mathrm{p}, 2 \mathrm{~s}}+n_{\mathrm{H}, 3 \mathrm{~d}} A_{3 \mathrm{~d}, 2 \mathrm{p}}},
$$

where we approximate $\phi_{v, \mu}^{3 \mathrm{~s}, 2 \mathrm{p}} \approx \phi_{v, \mu}^{3 \mathrm{p}, 2 \mathrm{~s}} \approx \phi_{v, \mu}^{3 \mathrm{~d}, 2 \mathrm{p}}$. (see also [ㅁ] ]). Similarly, we define the polarization degree for the spatially integrated Stokes parameters as

$$
\tilde{\Pi}_{0}=\frac{\int Q_{v, 0} \mathrm{~d} v \mathrm{~d} z}{\int I_{v, 0} \mathrm{~d} v \mathrm{~d} z}
$$

Note that in order to describe the shock energy loss, we use the shock jump conditions derived by [1[7] and solve the ionization structure of hydrogen based on the jump conditions. For the numerical calculation, we fix the ionization degree of far upstream region as $\chi_{0}=0.5$ and the ratio of downstream electron temperature to downstream proton temperature as $\beta=0.1$ (see [ए]6] for detail).

\section{Results and Conclusions}

Figure $\square$ shows the spatial profile of polarization degree of $\mathrm{H} \alpha, \Pi_{0}(z)$, for the energy loss rates of $\eta=(0,0.3,0.5)$ with given $k_{\mathrm{B}} T_{\mathrm{p}}=12 \mathrm{keV}$, where $T_{\mathrm{p}}$ is the downstream proton temperature. A

\footnotetext{
${ }^{1}$ In particular, the former assumption may be invalid because the conversion of $\operatorname{Ly} \beta$ to $\mathrm{H} \alpha$ corresponds to the scattering of electromagnetic wave that can yield a polarized light in general. We will investigate this in future work.
} 


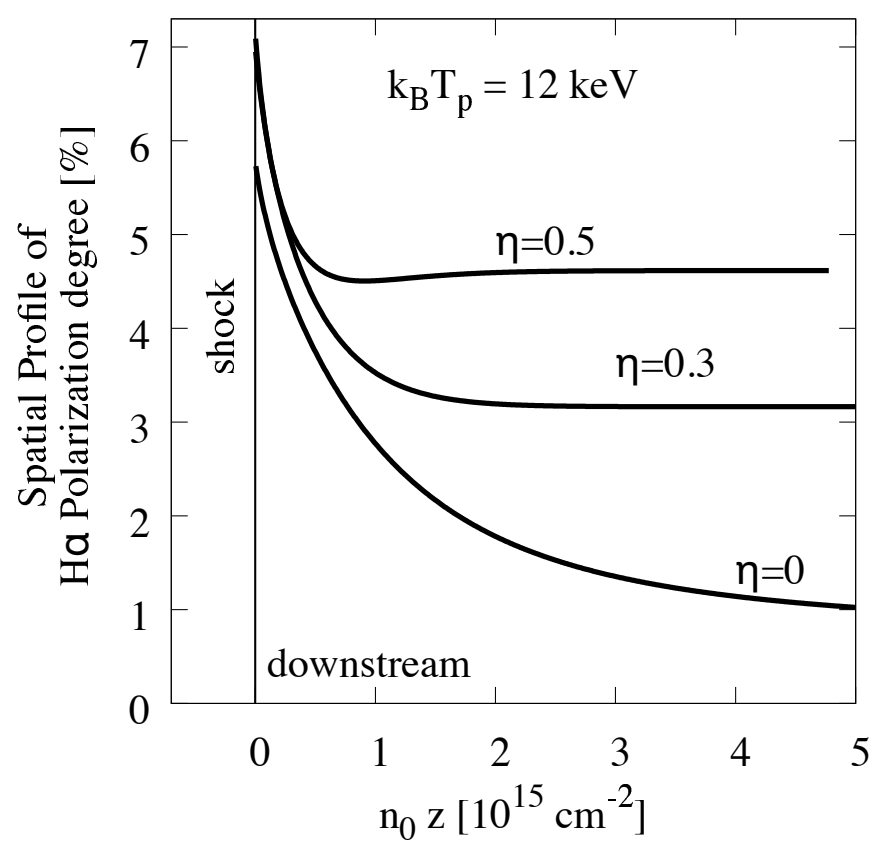

Figure 1: Spatial profile of polarization degree of $\mathrm{H} \alpha, \Pi_{0}(z)$, for $\eta=(0,0.3,0.5)$ with given $\beta=0.1$, $\chi_{0}=0.5$ and $k_{\mathrm{B}} T_{\mathrm{p}}=12 \mathrm{keV}$, where $T_{\mathrm{p}}$ is the downstream proton temperature. The shock is located at $z=0$. The spatial profile is normalized by the number density of far upstream region, $n_{0}$.

larger shock energy loss due to the production of non-thermal particles results in a larger polarization degree of $\mathrm{H} \alpha$ because the aniosotropy of collisions between hydrogen atoms and downstream protons becomes stronger. This result is consistent with [12]. The energy loss rate can be evaluated from the spatial profile, although the $\mathrm{H} \alpha$ emission from the far downstream region becomes fainter and difficult to observe. It may be also difficult to obtain the spatially resolved profile by current observations that need roughly better than 0.1 arcsecond angular resolution.

Figure $\square$ shows the spatially unresolved polarization degree of $\mathrm{H} \alpha, \tilde{\Pi}_{0}$, as function of downstream proton temperature for fixed values $\eta=(0,0.3,0.5)$. The energy loss rate $\eta$ can be evaluated even if there are no spatially resolved polarization measurements. In comparison with observations of SNR SN $1006\left(k_{\mathrm{B}} T_{\mathrm{p}} \approx 9.87 \pm 0.68\right.$ [ए5]]; $\tilde{\Pi}_{0}=2.0 \pm 0.4$ [ए3] $)$, we find that the shock energy loss rate of SNR SN 1006 may be as sizable as $\eta \sim 0.1$. Note that the polarization degree would depend on the electron temperature and ionization degree of far upstream region that are fixed in this paper for simplicity. Moreover, it is still unclear whether the spatially unresolved polarization degree observed by [[13] is affected by $\mathrm{H} \alpha$ emissions from upstream region originating from the $\mathrm{Ly} \beta-\mathrm{H} \alpha$ conversion. In order to obtain more strict constraint about $\eta$, we will investigate them in a forthcoming paper.

\section{Acknowledgements}

This work is partially supported by JSPS KAKENHI grant no. JP18H01245. JML was supported by the Guest Investigator Grant HST-GO-13435.001 from the Space Telescope Science 


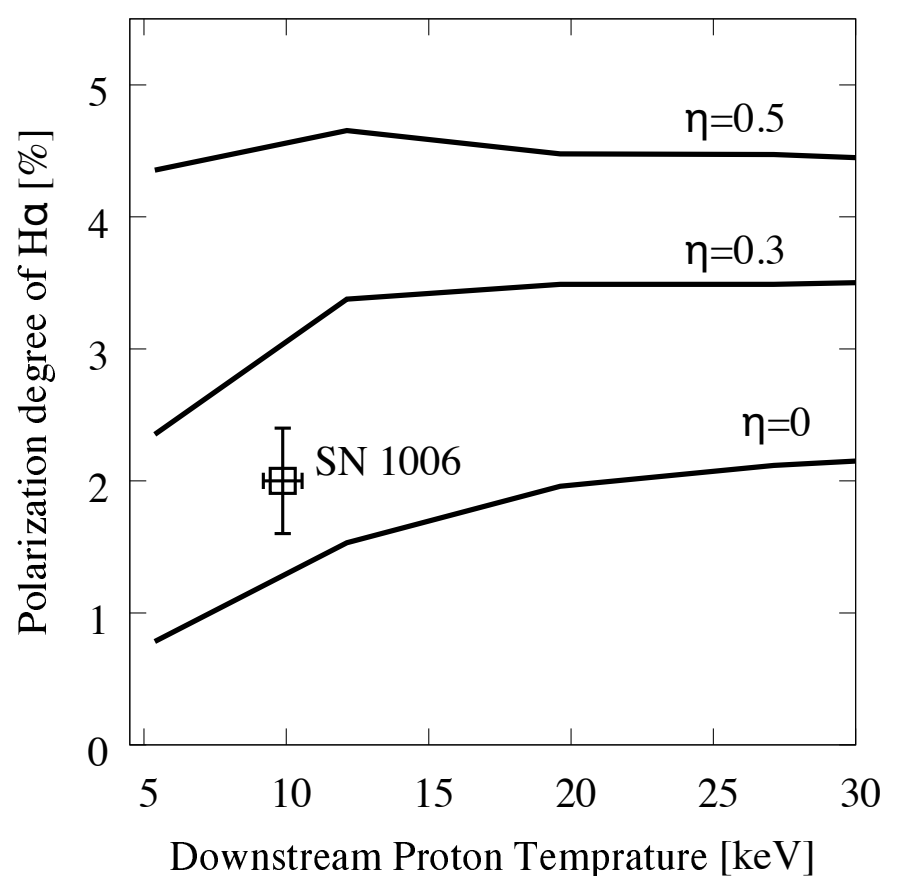

Figure 2: Spatially unresolved polarization degree of $\mathrm{H} \alpha, \tilde{\Pi}_{0}$, as function of the downstream proton temperature for fixed values $\eta=(0,0.3,0.5)$. The actual observation data of SNR SN 1006 is also displayed (from [피] and [ㅍ]]).

Institute and by the NASA Astrophysics Theory Program (80HQTR18T0065), as well by Basic Research Funds of the CNR. 


\section{References}

[1] A. Boulares and D. P. Cox, Galactic Hydrostatic Equilibrium with Magnetic Tension and Cosmic-Ray Diffusion, ApJ 365 (1990) 544.

[2] A. A. Goodman, P. J. Benson, G. A. Fuller and P. C. Myers, Dense cores in dark clouds. VIII Velocity gradients, ApJ 406 (1993) 528 .

[3] R. M. Crutcher, Magnetic Fields in Molecular Clouds: Observations Confront Theory, ApJ 520 (1999)706.

[4] S.-i. Inutsuka, Present-day star formation: From molecular cloud cores to protostars and protoplanetary disks, Progress of Theoretical and Experimental Physics 2012 (2012)01A307.

[5] C. F. McKee and J. P. Ostriker, A theory of the interstellar medium: three components regulated by supernova explosions in an inhomogeneous substrate., ApJ 218 (1977) 148.

[6] V. L. Ginzburg and S. I. Syrovatskii, The Origin of Cosmic Rays. 1964.

[7] J. P. Hughes, C. E. Rakowski and A. Decourchelle, Electron Heating and Cosmic Rays at a Supernova Shock from Chandra X-Ray Observations of 1E 0102.2-7219, ApJL 543 (2000) L6] [astro-ph/0007032].

[8] E. A. Helder, J. Vink, C. G. Bassa, A. Bamba, J. A. M. Bleeker, S. Funk et al., Measuring the Cosmic-Ray Acceleration Efficiency of a Supernova Remnant, Science 325 (2009) 719 [0906.4553].

[9] L. Hovey, J. P. Hughes, C. McCully, V. Pandya and K. Eriksen, Constraints on Cosmic-ray Acceleration Efficiency in Balmer Shocks of Two Young Type Ia Supernova Remnants in the Large Magellanic Cloud, ApJ 862(2018) 148 [1109.08273].

[10] J. Shimoda, T. Inoue, Y. Ohira, R. Yamazaki, A. Bamba and J. Vink, On Cosmic-ray Production Efficiency at Supernova Remnant Shocks Propagating into Realistic Diffuse Interstellar Medium, Apd $803(2015) 98$ [11412.2874].

[11] E. A. Helder, J. Vink, A. Bamba, J. A. M. Bleeker, D. N. Burrows, P. Ghavamian et al., Proper motions of $H \alpha$ filaments in the supernova remnant $R C W$ 86, MNRAS 435 (2013) 910 [11306.3994].

[12] J. Shimoda, Y. Ohira, R. Yamazaki, J. M. Laming and S. Katsuda, Polarized Balmer line emission from supernova remnant shock waves efficiently accelerating cosmic rays, MNRAS 473 (2018) 1394 [1709.04966].

[13] W. B. Sparks, J. E. Pringle, R. F. Carswell, K. S. Long and M. Cracraft, Discovery of Polarized Line Emission in SN 1006, ApJL815(2015) L9] [1511.06012].

[14] H. Kleinpoppen and E. Kraiss, Cross Section and Polarization of the $H_{\alpha}$ Line by Electron Impact Excitation, Phys. Rev. Letter 20(1968) 361.

[15] P. Ghavamian, J. Raymond, R. C. Smith and P. Hartigan, Balmer-dominated Spectra of Nonradiative Shocks in the Cygnus Loop, RCW 86, and Tycho Supernova Remnants, ApJ547(2001) 995 astro-ph/0010496.

[16] J. Shimoda and J. M. Laming, Radiative transfer of hydrogen lines from supernova remnant shock waves: contributions of $2 s$-state hydrogen atoms, MNRAS 485 (2019) 5453 [11903.01262].

[17] E. Cohen, T. Piran and R. Sari, Fluid Dynamics of Semiradiative Blast Waves, ApJ 509(1998) 717 astro-ph/9803258]. 